\title{
HIDROGEL PARA IMPRESSÃO 4D
}

Renato Maffei Bastos Gomes (renato.maffei@gmail.com) - Centro de Engenharia, Modelagem e Ciências Sociais Aplicadas, Universidade Federal do ABC.

Silvia Lenyra Meirelles Campos Titotto (titotto@gmail.com) - Centro de Engenharia, Modelagem e Ciências Sociais Aplicadas, Universidade Federal do ABC.

\section{RESUMO}

A realidade da impressão $3 D$ e sua evolução para impressão 4D se mostram como alternativas para produção de tecnologias. Algumas pesquisas nas fronteiras do conhecimento em andamento em grandes centros de pesquisa no exterior, como o Wyss Institute da Harvard University, recentemente têm feito uso de hidrogéis para avanços em impressão 4D de forma a conseguirem mimetizar, sob estímulos ambientais, movimentos naturais de sistemas biológicos. O presente trabalho, que mostra resultados preliminares de pesquisa em andamento, consiste no estudo de materiais hidrogéis e suas possibilidades de aplicação por meio de impressão 4D, descrevendo os principais fatores relacionados a este método e as propriedades do hidrogel que justificam seu uso neste contexto. Esta investigação tem a intenção de, inicialmente, estabelecer a constituição e as variações dos materiais hidrogéis e, posteriormente, determinar aplicações que envolvam estes materiais e métodos, visando uma descrição suficiente para esclarecer a função do hidrogel e das técnicas de impressão utilizadas nas aplicações avaliadas. A partir dos resultados preliminares, conclui-se que os principais fatores referentes a impressão $4 D$ são os materiais, os estímulos ambientais, o design e os instrumentos, sendo necessários avanços relacionados aos mecanismos e materiais utilizados nas impressões.

Palavras chave: impressão 4D; impressão 3D; hidrogel; biomimética; aplicações

Área: Ferramentas e métodos de desenvolvimento de produtos e serviços 


\section{INTRODUÇÃO}

\subsection{Contextualização}

A evolução de técnicas para elaboração de tecnologias tem possibilitado maior rapidez e eficiência na produção de dispositivos cada vez mais complexos e inovadores. A disposição de diversos materiais, arquiteturas, máquinas, métodos matemáticos e métodos computacionais sofisticados incentiva a fabricação de objetos por meio de impressoras.

A manufatura aditiva ou impressão 3D tem estado cada vez mais presente nos processos de fabricação e no mercado industrial. A tecnologia de impressão que consiste na deposição de materiais em camadas está no mercado desde o final da década de 1980, mas os recentes avanços tecnológicos, a partir do ano 2000, nas áreas de materiais e eletrônica impulsionaram seu desenvolvimento e deram origem à manufatura aditiva como é conhecida atualmente, também chamada de prototipagem rápida (MARQUES, 2014).

O estabelecimento da impressão 3D tem permitido a produção de objetos tridimensionais com o uso de materiais como plásticos, metais e materiais cerâmicos. A evolução dos mecanismos e a possibilidade de impressão com multimateriais e materiais com automontagem viabilizam a impressão 4D, a qual inclui a dimensão do tempo aos objetos impressos, que podem se modificar de acordo com estímulos do ambiente (CHOI et al., 2015).

Figura 1. Impressora 4D em atividade. Fonte: Wyss Institute (2016)

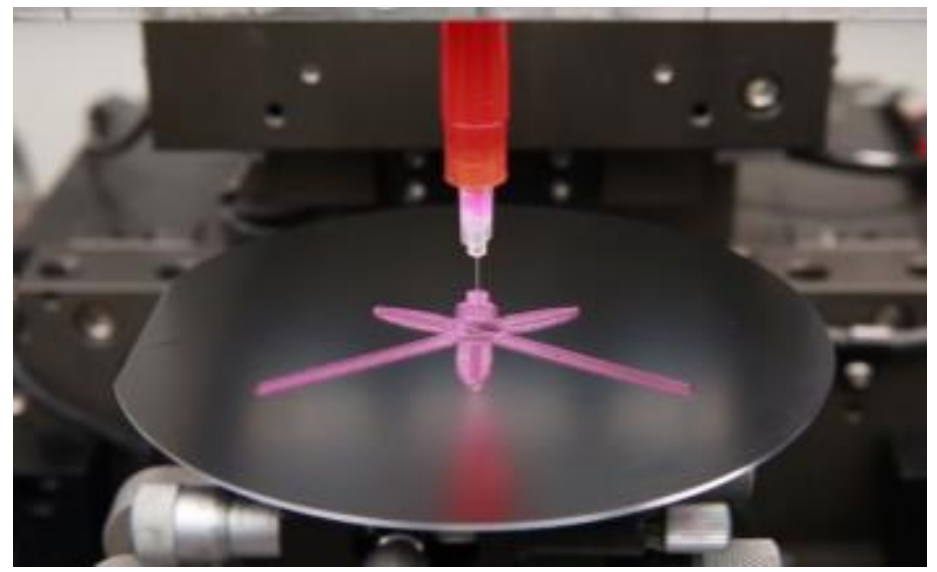

\subsection{Problema}

A investigação das possíveis aplicações na área de impressão 3D e 4D possui grande relevância para que os resultados de pesquisas possam se converter em dispositivos úteis. Neste sentido, destaca-se o hidrogel como material com potencial para produção destas tecnologias.

Ao avaliar as propriedades do hidrogel, pode-se verificar que sua capacidade de alteração de comportamento em resposta a fatores externos (OTTENBRITE et al., 2010) justifica seu uso para impressão 4D. O estudo destas propriedades e das influências que podem exercer nas funções de um produto que contém hidrogel é fundamental para a caracterização das aplicações, sendo necessário definir as alterações possíveis e desejadas do material ao longo do funcionamento do dispositivo.

Por meio do estudo das propriedades do hidrogel, das possibilidades atuais e das perspectivas 
para o futuro de aplicações na área de impressão 3D e 4D, o tema desta pesquisa é a definição da função do hidrogel quando inserido nestas tecnologias, buscando formas viáveis e efetivas, além da previsão da expectativa gerada por cada aplicação.

\subsection{Justificativa}

A pesquisa relacionada a impressão 4D é ainda incipiente no Brasil e na América Latina, e tem sido tema de pesquisas em grandes universidades do exterior como a Harvard University, o Massachusetts Institute of Technology (MIT) e o Korea Institute of Science and Technology. Por isso, entende-se que o estudo de materiais, formas, estruturas e condições relacionadas aos métodos de impressão 3D e 4D é oportuno e possibilita avanços tecnológicos comuns a universidades e países de destaque científico, levando em consideração que materiais hidrogéis possuem muitas possibilidades de aplicação e evolução para o campo, que tem potencial para revolucionar a criação de tecnologias e a forma como objetos são produzidos, conforme mostram Tibbits (2017) e Tibbits e seus colegas (2014).

\section{OBJETIVOS}

Este trabalho apresenta resultados preliminares de pesquisa em andamento que tem como objetivo geral o estudo de materiais hidrogéis e suas possibilidades de aplicação por meio de impressão 4D. De forma específica, tem-se buscado estabelecer a constituição e padrões de hidrogel, estudar mecanismos de impressão 4D, buscar inspirações em sistemas biológicos naturais, determinar aplicações e analisar impactos destas possíveis aplicações.

\section{METODOLOGIA}

\subsection{Métodos}

O método de pesquisa consiste no acompanhamento contínuo de publicações referentes à área estudada, partindo de pesquisa sucessiva de técnicas e resultados obtidos por meio de impressão 3D e 4D. Busca-se estabelecer a constituição e as variações de materiais hidrogéis, com foco nas características estruturais e relacionadas a alterações da forma a partir de estímulos do ambiente.

A análise dos dados coletados visa a determinação de aplicações para o hidrogel por meio de impressão, incluindo possíveis impactos destas. A partir das propriedades estudadas do material e com base nos resultados relatados na literatura, a intenção é estabelecer uma área de interesse, como as de eletrônicos, têxteis ou dispositivos médicos, destacadas por Gladman et al. (2016), e analisar as principais tecnologias existentes na área, com foco naquelas em que o hidrogel poderia, de alguma forma, contribuir para um melhor desempenho. Este processo deve se repetir para as outras áreas mencionadas e, para cada caso, deve ser feita uma descrição dos fatores envolvidos na aplicação, principalmente a contribuição do hidrogel e dos métodos de impressão envolvidos.

\subsection{Forma de análise}

As formas de análise têm como base a descrição dos principais aspectos necessários para conceber pelo menos uma aplicação em cada uma das seguintes áreas: eletrônicos, têxteis e dispositivos médicos. Esta descrição deve ser suficiente para esclarecer a função do hidrogel e das técnicas de impressão utilizadas nos processos relatados, o que necessita de detalhamento dos materiais, das técnicas, das características fundamentais e de comparações, visando as possibilidades de aplicação de forma a fornecer informações essenciais para o estudo e 
produção destas.

\subsection{Etapas}

A primeira parte da pesquisa, a qual refere-se ao esforço preliminar presente neste trabalho, consiste na investigação dos fundamentos básicos que possibilitam iniciar o estudo de aplicações. Partindo de uma revisão bibliográfica, investiga-se os métodos de impressão 3D e 4D e as propriedades do hidrogel. Os conteúdos estudados servem como base para o estudo preparatório das aplicações já existentes e daquelas que se deseja definir, seguido de nova revisão bibliográfica, por meio da qual deve-se acompanhar as novidades do tema abordado e dar encaminhamento à segunda parte da pesquisa.

A segunda parte do projeto, que deve ser iniciada após a conclusão deste trabalho, consiste na determinação de aplicações e descrição destas a partir das informações coletadas na primeira parte. Inicialmente, serão listadas as ideias de aplicações consideradas viáveis e em seguida, realizando nova revisão bibliográfica com propósito de atualização, serão definidas e descritas as aplicações mais relevantes nas respectivas áreas de interesse.

\section{RESULTADOS}

Dentre os resultados preliminares, destaca-se a apresentação dos principais fatores relacionados às técnicas de impressão 3D e 4D e a definição das propriedades de materiais hidrogéis que possam ser utilizadas, em conjunto com as técnicas descritas, como apoio para a posterior determinação e especificação de aplicações e previsão de seus impactos.

\subsection{Métodos 3D e 4D}

De acordo com Choi e seus colegas (2015), impressão 3D é definida como um método de manufatura aditiva para fabricação de estruturas 3D pela disposição de materiais em camadas dependente de um design pré-determinado. A impressão 4D é similar à impressão 3D, mas adiciona uma quarta dimensão, o tempo, como ilustra a Figura 2. Assim, permite-se que uma estrutura mude sua forma ou função com o tempo em resposta a estímulos como pressão, temperatura, vento, água e luz.

Figura 2. Esquema de conceitos 1D, 2D, 3D, 4D. Fonte: Choi et al. (2015)

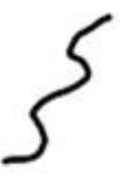

1D

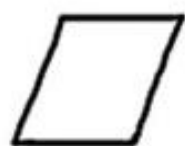

2D

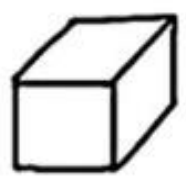

$3 \mathrm{D}$

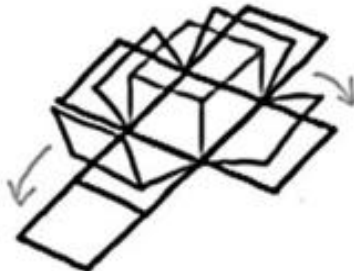

4D

A presença de design inteligente e materiais inteligentes é a principal diferença, em termos de aspectos técnicos, da impressão 4D em relação à impressão 3D. Para construir estruturas 3D, materiais como plásticos, metais e cerâmicas são usados. Porém, a maioria desses materiais não é aplicável a impressão 4D por conta da sua falta de reação a estímulos externos. Para resolver este problema, é necessário o uso de materiais projetados, multimateriais ou materiais com automontagem, que podem ser polímeros, materiais com degradação ou deformação própria e outros materiais conhecidos ou a serem desenvolvidos no sentido de gerar mudanças na forma, 
cor ou função após a disposição de suas camadas por meio da impressão. A seguir, apresenta-se a influência de determinados fatores externos sobre o comportamento do material utilizado.

No caso da impressão 4D, a função desempenhada pelo material ao longo de sua utilização é dependente do estímulo ambiental a que ele é submetido, por meio do qual altera-se propriedades específicas no nível atômico e molecular, o que pode se manifestar nas características macroscópicas da estrutura geral. Choi et al. (2015) exemplifica esta influência com casos de materiais que respondem a variações de temperatura e presença de luz.

Materiais responsivos a temperatura, como polímeros de memória de forma e ligas, possuem a habilidade de se contrair ou expandir em resposta a alterações na temperatura. No caso dos polímeros, a estrutura formada deve se alterar de acordo com as variações de temperatura, e quando atinge-se a temperatura crítica para mudança de forma, a estrutura deformada retorna à configuração original.

Em determinados materiais, pode haver resposta a luz, como irradiação UV ou luz solar, que pode gerar alteração na cor do objeto 3D. Isto pode ocorrer, por exemplo, em cadeias de polímeros responsivos a radiação UV que incluem azo-compostos, compostos químicos de grupo funcional $\mathrm{R}-\mathrm{N}=\mathrm{N}-\mathrm{R}$ '. A mudança de cor ocorre pela alteração de compostos da cadeia polimérica, como mostra a Figura 3.

Figura 3. Esquema de mudanças na estrutura do polímero em resposta a luz. Fonte: Choi et al. (2015)

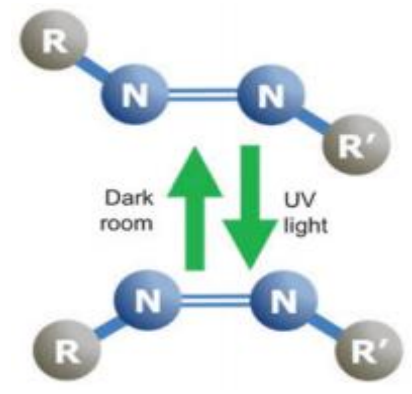

Outro importante fator externo é a presença de água. Isto é demonstrado por Gladman et al. (2016), que produziram estruturas 4D impressas com a utilização de estruturas compostas por hidrogel que mudam de forma após imersão em água. As formas estabelecidas são inspiradas em estruturas naturais como plantas e buscam imitar seu comportamento, ou seja, utiliza-se de biomimética. A Figura 1 (seção Contextualização) mostra o caminho de impressão estabelecido, e a Figura 4 mostra parte dos resultados obtidos.

Figura 4. Estrutura composta por hidrogel após submersão em água. Fonte: Gladman et al. (2016)

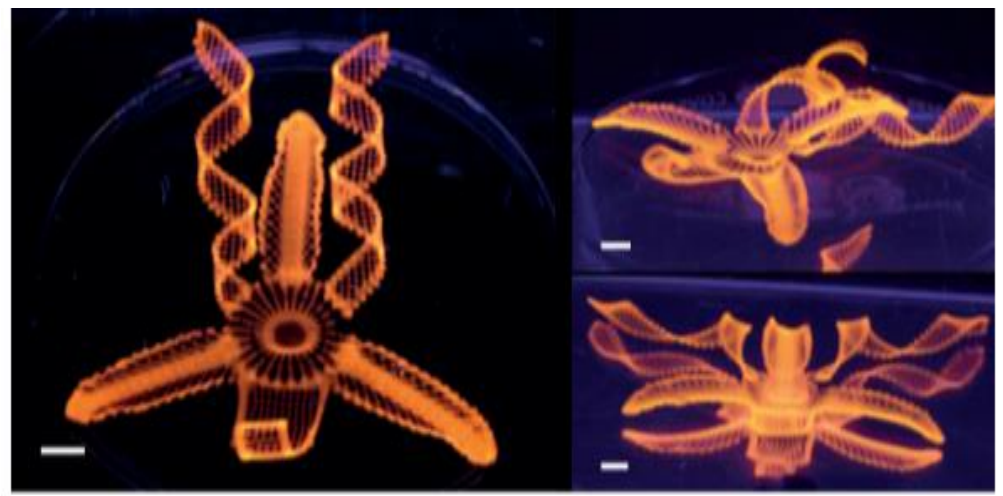


Foi mostrado que a aplicabilidade do material depende do estímulo ambiental para impressão 4D, mas para que a impressão seja feita de forma satisfatória, é fundamental definir o design do item a ser impresso. As alterações no material devem ser controladas pelo design do produto, de forma a planejar e prever as formas e comportamentos que este deve possuir ao longo de seu funcionamento (CHOI et al., 2015), a partir das condições ambientais.

Para que o design seja estabelecido e executado adequadamente, é essencial ter à disposição instrumentos efetivos, o que inclui métodos de programação eficientes para a situação, formas de simular os resultados propostos, procedimentos matemáticos que validem o projeto e a própria impressora 3D, cujas capacidades e limitações devem ser consideradas, principalmente para a impressão 4D, que envolve maior complexidade em grande parte dos aspectos observados.

\subsection{Hidrogel}

Como mencionado anteriormente, certos materiais poliméricos possuem potencial de uso em impressão 4D devido à alteração de determinados aspectos a partir de estímulos ambientais. Este é o caso de materiais hidrogéis, exemplificados visualmente na Figura 5 e descritos a seguir.

Figura 5. Exemplo de hidrogel. Fonte: Technion - Israel Institute of Technology (2015)

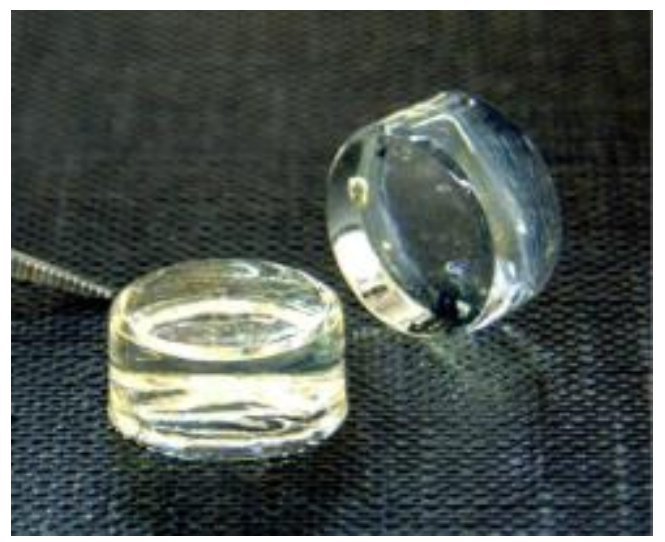

Ottenbrite e seus colegas (2010) definem hidrogéis como uma classe de polímeros de ligação cruzada que, devido à sua natureza hidrofílica, podem absorver grandes quantidades de água. Assim, o hidrogel se torna composto por sólido (polímero) e líquido (água). A ligação cruzada refere-se à ligação entre cadeias poliméricas.

Em estado inchado, estes materiais oferecem, de forma significante, estabilidade física, química e mecânica. As propriedades finais do hidrogel são determinadas tanto pelo polímero específico quanto pela composição do composto em termos da razão entre quantidade de polímero e quantidade de água absorvida.

Têm sido desenvolvidos diversos tipos de géis poliméricos que respondem a mudanças em seus arredores, o que é interessante para as áreas de materiais inteligentes, incluindo biomimética. Nesta área, tem-se como exemplo o trabalho de Gladman e seus colegas (2016), citado anteriormente, que usa tinta de hidrogel composta por fibrilas de celulose rígidas incorporadas em uma matriz de acrilamida macia, que imita a composição de paredes celulares de plantas e que gera os resultados mostrados na Figura 4 após imersão em água. Gladman et al. mencionam as possibilidades de tecnologias que podem ser promovidas com 
estes resultados, incluindo aplicações nas áreas de têxteis, eletrônicos e dispositivos médicos, as quais serão melhor investigadas no prosseguimento desta pesquisa de hidrogel para impressão 4D.

\section{CONCLUSÕES}

Temos que os principais fatores referentes à impressão 4D, que devem ser priorizados na avaliação das aplicações, são os materiais, os estímulos ambientais, o design e os instrumentos. Os fatores externos ao objeto impresso são mais relevantes na impressão 4D do que na 3D, enquanto os materiais, o design e os instrumentos são diferentes na comparação dos dois métodos devido à diferença de complexidade em cada situação, já que os métodos 4D exigem técnicas mais sofisticadas.

Enquanto as aplicações de impressão 3D envolvem eletrônicos, brinquedos, têxteis, automóveis, dispositivos médicos e outros, as aplicações de impressão 4D envolvem todas as aplicações da impressão 3D visando mudanças de configuração de forma dinâmica, sendo fundamental a escolha correta do material para que os resultados sejam adequados (CHOI et al., 2015). Choi et al. (2015) e Tibbits (2014) confirmam que, apesar de serem muitas as possibilidades na área de impressão 4D, necessita-se de avanços quanto aos mecanismos e materiais utilizados nas impressões para que seja possível concretizá-las de forma satisfatória.

Considerando esta grande variedade de aplicações, observa-se que os polímeros são materiais de grande importância neste contexto, sendo o hidrogel útil para situações em que suas principais propriedades sejam favoráveis, ou seja, para casos em que sua mudança de forma perante estímulos do ambiente ou seu uso como revestimento seja apropriado e não prejudique as funcionalidades do dispositivo em que o material faz parte, já que possui grande capacidade de absorção de água. Outro fator importante é o custo de produção, que deve ser analisado ao se optar pelo uso de um material, em conjunto com suas propriedades.

\section{REFERÊNCIAS}

CHOI, J. et al. 4D Printing Technology: A Review. 3D Printing and Additive Manufacturing. December 2015, 2(4): 159-167, 2015.

GLADMAN, A. S. et al. Biomimetic 4D printing. Nature Materials. 15: 413-418, 2016.

MARQUES, K. Manufatura aditiva: o futuro do mercado industrial de fabricação e inovação. Portal da Escola de Engenharia de São Carlos, Universidade de São Paulo, 2014. Disponível em:

<http://www.eesc.usp.br/portaleesc/index.php?option=com_content\&view=article\&id=1934: manufatura-aditiva-o-futuro-do-mercado-industrial-de-fabricacao-einovacao\&catid=115\&Itemid=164>. Acessado em 24/05/2017.

OTTENBRITE, R. M. et al. Biomedical Applications of Hydrogels Handbook. SpringerVerlag New York, 2010.

Technion - Israel Institute of Technology. Biomaterials \& Regenerative Medicine, 2015. Disponível em: <http://brm.technion.ac.il/gallery/>. Acessado em 26/05/2017.

TIBBITS, S. Multi-material Shape Change. Architectural Design. January/February 2014, 84(1): 116-121, 2014. 
TIBBITS, S. Renewable Future. 3D Printing and Additive Manufacturing March 2017, 4(1): $1-1,2017$.

TIBBITS, S. et al. 4D printing and universal transformation. Acadia 2014 - Design Agency: 539-548, 2014.

WEGST, U. G. K. et al. Bioinspired structural materials. Nature Materials, 14(1): 23-36, 2015 .

Wyss Institute. Novel 4D printing method blossoms from botanical inspiration, 2015. Disponível em: <https://wyss.harvard.edu/novel-4d-printing-method/>. Acessado em 24/05/2017. 